\title{
Diversity studies on arbuscular mycorrhizal fungi in vegetable crop plants of Goa, India
}

\section{Dessai SA $*$ and Rodrigues BF}

Department of Botany, Goa University, Goa 403206 India.

Dessai SA, Rodrigues BF 2012 - Diversity studies on arbuscular mycorrhizal fungi in vegetable crop plants of Goa, India. Plant Pathology \& Quarantine 2(1), 87-101, doi 10.5943/ppq/2/2/1

This study was conducted to assess the arbuscular mycorrhizal fungal diversity associated with different vegetable crop plants cultivated in Goa. Fifty one AM fungal species were recovered from rhizosphere soil samples of 27 vegetable crop plants belonging to 10 families from 10 different agricultural sites of Goa during 2008-2009. Glomus (26) was the dominant genus followed by Acaulospora (16), Gigaspora (4) and Scutellospora (5) with species number given in parentheses. The maximum spore density was recorded in Cansaulim (1015.01 spores $100 \mathrm{~g}^{-1} \mathrm{soil}$ ) and minimum was reported in Pernem (394.01spores $100 \mathrm{~g}^{-1}$ soil). The highest number of arbuscular mycorrhizal spores was found in Zea mays (95.33 spores $100 \mathrm{~g}^{-1}$ soil) in Taleigao and least was recovered in $A$. virdis (12.33 spores $100 \mathrm{~g}^{-1}$ soil) in Taleigao. Acaulospora scrobiculata was recorded in all ten sites in 24 vegetables and was the dominant species in six sites. Species richness was maximum in Netravali $\left(28\right.$ species $^{\text {-site }}$ ). Simpsons and Shanon-Wiener Diversity Indices of arbuscular mycorrhizal fungi were highest in Netravali respectively.

Key words - Agricultural sites - dominant genus - rhizosphere soil samples - species richness spore density

\section{Article Information}

Received 30 May 2012

Accepted 3 July 2012

Published online 30 July 2012

*Corresponding author: Bernard Felinov Rodrigues - e-mail - felinov@gmail.com

\section{Introduction}

Arbuscular mycorrhizal (AM) fungi belonging to the phylum Glomeromycota (Wang \& Qiu 2006) are vital components of almost all terrestrial ecosystems, forming a mutualistic symbiosis with roots of $\mathrm{ca} 80 \%$ of vascular plants (Smith et al. 2003), including vegetable crop plants and agronomically important species (Harrier \& Watson 2004). As obligate symbionts AM fungi are dependent upon host plants for fixed carbon (Rezaee et al. 2007). In exchange plants in agricultural and natural ecosystems receive various benefits from AM fungi viz., improvement in the uptake of diffusion limited macronutrients such as $\mathrm{P}$ (Rivera et al. 2005) and other minerals such as $\mathrm{K}, \mathrm{Fe}, \mathrm{Cu}, \mathrm{Ca}, \mathrm{Mg}$ and $\mathrm{Zn}$ (George 2000; Liu et al. 2002; Yaseen et al. 2011), increase in the ability to overcome biotic and abiotic stresses (Pojo \& Azcon 2007) such as protection of plants against soil-borne diseases, water relations and soil aggregation and enhancement in crop growth and yield (Douds et al. 2005). Application of AM fungi has resulted in enhanced productivity in cereals, pulses, oilseeds, vegetables and ornamental plants (Boddington \& Dodd 2000).

AM fungal diversity shows variation dependent upon plant species (Blaszkowski 1994). In particular, AM fungi are important in organic and sustainable farming systems that rely on biological processes rather than agrochemicals (Harrier \& Watson 2004), thus offering a great potential for sustainable agricultural system (Khalil et al. 1992). A better understanding of the field study based on 


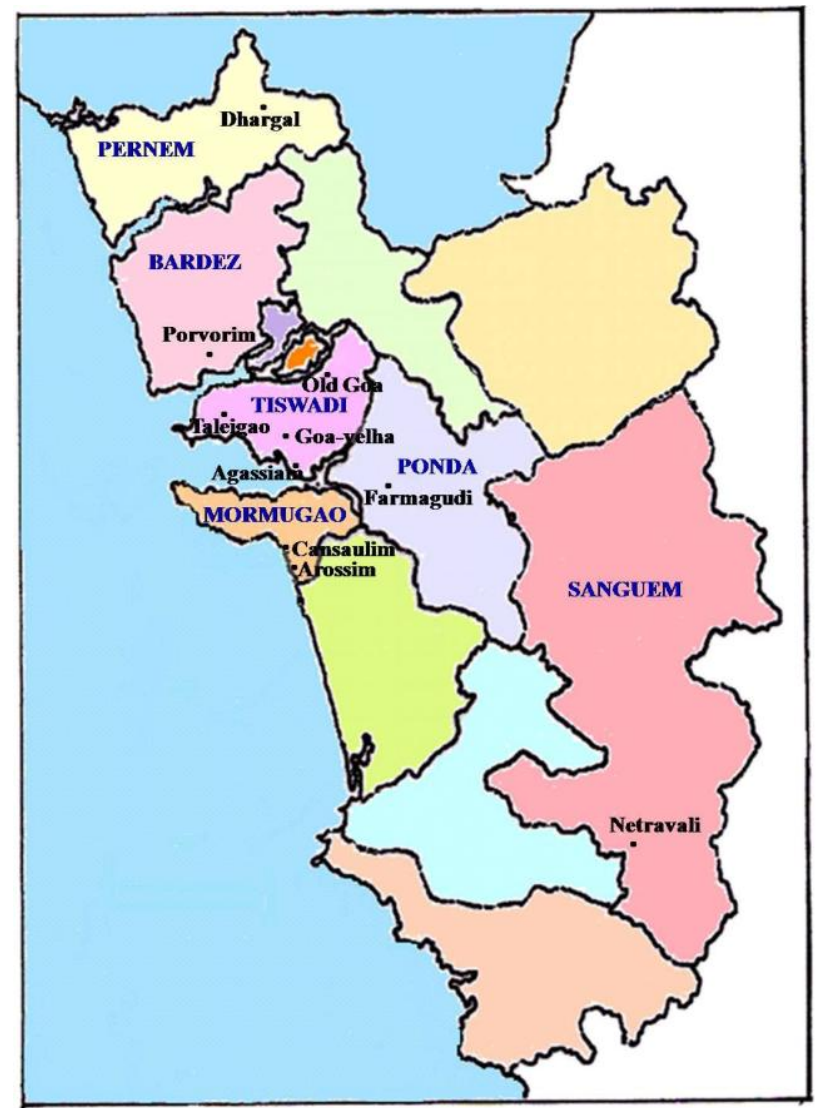

Fig. 1 - Map of Goa showing study sites

AM fungal diversity associated with agronomic crops is necessary. The objective of this study is to assess the AM fungal diversity associated with different vegetable crop plants cultivated in Goa. AM fungal colonization and soil analysis of rhizosphere soils of the agricultural fields was also determined.

\section{Materials and methods}

Rhizosphere soil and root samples of 27 cultivated vegetable crops were collected from 10 different sites viz., Cansaulim (S-I), Dhargal (S-II), Old-Goa (S-III), Taleigao (S-IV), Agassim (S-V), Farmagudi (S-VI), Porvorim (S-VII), Netravali (S-VIII), Arossim (S-IX), and Goa-Velha (S-X), during 2008-2009 (Fig. $1)$. The climate is dry tropical, warm and humid with loamy-sandy soil. Mean temperature range from $20^{\circ} \mathrm{C}-35^{\circ} \mathrm{C}$, with an average rainfall of $2500 \mathrm{~mm}$.

Soil samples were collected from a depth of $0-25 \mathrm{~cm}$ from the selected study sites. Soil $\mathrm{pH}$ was measured by $\mathrm{pH}$ meter in a $1: 1 \mathrm{H}_{2} \mathrm{O}$ solution (LI 120 Elico, India). Electrical Conductivity (EC) was measured using Conductivity meter (CM-180 Elico, India).
Organic carbon (C) and available phosphorus (P) were analyzed using the standard soil analysis techniques viz., Walkley \& Black (1934) rapid titration method and Bray \& Kurtz method (1945), respectively. Available potassium (K) was estimated by ammonium acetate method (Hanway \& Heidal 1952) using flame photometer (Systronic 3292). Available zinc $(\mathrm{Zn})$, copper $(\mathrm{Cu})$, manganese $(\mathrm{Mn})$ and iron $(\mathrm{Fe})$ were quantified by DTPA- $\mathrm{CaCl}_{2-}$ TEA method (Lindsay \& Norwell 1978) and boron (B) by the hot water soluble method (Berger \& Truog 1939) using Atomic Absorption Spectrophotometer (AAS 4139).

Root samples were processed for AM fungal colonization using the method of Koske \& Gemma (1989). The stained roots were examined using an Olympus research microscope BX 41 (100X - 1000X) for AM fungal structures and percent root length colonization was determined following the slide method (Giovannetti \& Mosse 1980). AM fungal spores were isolated by wet sieving and decanting method (Gerdemann \& Nicolson 1963).

Intact and unparasitized spores were used for the quantification of spore density and 
Table 1 Soil chemical analysis in the agricultural sites in Goa.

\begin{tabular}{|c|c|c|c|c|c|c|c|c|c|c|}
\hline \multirow[t]{2}{*}{ Sites } & \multirow[t]{2}{*}{ pH } & \multirow{2}{*}{$\begin{array}{c}\mathbf{E C} \\
\mathbf{m} / \\
\text { mhos/cm }\end{array}$} & \multicolumn{3}{|c|}{ Macro-nutrients } & \multicolumn{5}{|c|}{ Micro-nutrients (ppm) } \\
\hline & & & $\begin{array}{c}\text { Organic } \\
\text { Carbon } \\
\%\end{array}$ & $\begin{array}{l}\mathrm{P}_{2} \mathbf{O}_{5} \\
\text { ppm }\end{array}$ & $\begin{array}{c}\mathrm{K}_{2} \mathrm{O} \\
\mathrm{Kg} / \mathrm{Ha}\end{array}$ & $\mathbf{Z n}$ & $\mathbf{F e}$ & Mn & $\mathrm{Cu}$ & B \\
\hline Cansaulim & 6.8 & 0.577 & 0.35 & 29.93 & 291.2 & 0.84 & 99.36 & 18.85 & 0.50 & 0.23 \\
\hline Dhargal & 5.7 & 0.156 & 2.50 & 13.30 & 896.0 & 1.45 & 63.84 & 11.52 & 3.42 & 0.72 \\
\hline Old-Goa & 6.1 & 0.231 & 1.83 & 9.97 & 884.8 & 2.23 & 110.26 & 13.55 & 1.51 & 0.47 \\
\hline Taleigao & 6.5 & 0.707 & 0.32 & 17.95 & 369.6 & 1.50 & 43.00 & 30.20 & 1.80 & 0.21 \\
\hline Agassim & 6.8 & 0.818 & 0.52 & 33.25 & 358.4 & 6.42 & 58.96 & 82.10 & 7.47 & 0.60 \\
\hline Farmagudi & 4.8 & 0.293 & 1.83 & 26.60 & 470.4 & 5.40 & 69.36 & 145.20 & 7.24 & 0.60 \\
\hline Porvorim & 5.6 & 0.210 & 0.28 & 29.93 & 67.2 & 0.29 & 117.14 & 28.38 & 1.23 & 0.17 \\
\hline Netravali & 7.0 & 0.592 & 1.74 & 73.16 & 963.2 & 2.89 & 71.28 & 93.88 & 3.31 & 0.33 \\
\hline Arossim & 7.0 & 0.527 & 0.48 & 33.25 & 694.4 & 3.04 & 64.42 & 20.04 & 0.72 & 0.27 \\
\hline Goa-Velha & 6.8 & 0.757 & 0.41 & 30.13 & 257.6 & 1.32 & 56.62 & 15.42 & 1.21 & 0.31 \\
\hline
\end{tabular}

taxonomy of AM fungi. The identification of AM spores was based on morphotaxonomic criteria using available literature (Schenck \& Perez 1990; Morton \& Benny 1990; Almeida \& Schenck 1990; Rodrigues \& Muthukumar 2009).

\section{Statistical analysis}

Data were statistically analyzed for standard deviation. Variation in AM fungal root colonization in relation to spore density was determined by Pearson's correlation coefficient using WASP (Web Based Agricultural Package) 2.0 to $\mathrm{P} \leq 0.05$ significance level.

Diversity studies were carried out for AM fungal species richness (species richness of AM fungi is the number of different species present in a particular site), species evenness (the measure of the relative abundance of the different species making up the richness of an area), Simpsons's Diversity Index (1-D) and Shannon-Wiener Diversity Index $(H)$ using PAST (Paleontological Statistics Software Package for Education and Data Analysis) version 2.14 (Hammer et al. 2001).

\section{Results}

Results of the soil analysis are shown in
Table 1. Soil $\mathrm{pH}$ was found to be acidic to neutral ranging from 4.8 to 7 . Available soil-P was found to be high, ranging from 9.97 to $73.16 \mathrm{ppm}$. Available K ranged from 67.2 to $963.2 \mathrm{Kg} / \mathrm{Ha}$. Micronutrients content $\mathrm{Fe}, \mathrm{Mn}$, $\mathrm{Zn}, \mathrm{Cu}$ and $\mathrm{B}$ was variable at the study sites.

Twenty two of the 27 vegetable crops undertaken for the study, showed AM colonization (Fig. 3 a, b). Maximum root colonization was observed in Zea mays $(68.33 \%)$ at S-IV and minimum in Lagenaria siceraria $(36 \%)$ at S-IX (Table 2). AM colonization was absent in five vegetable crops, viz., Amaranthus cruentus, A. viridis, Brassica rapa, Raphanus sativus and Spinacia oleraceae (Table 2) belonging to Amaranthaceae and Brassicaceae. Pearson's correlation coefficient for AM root colonization and spore density showed significance in all the sites.

Maximum spore density was recorded in S-I (1015.01 spores $100 \mathrm{~g}^{-1}$ soil) and minimum in S-II (394.01 spores100 $\mathrm{g}^{-1}$ soil) (Table 3). The highest number of AM spores was found in Z. mays (95.33 spores $100 \mathrm{~g}^{-1}$ soil) in S-IV, and the least recovered in $A$. viridis (12.33 spores $100 \mathrm{~g}^{-1}$ soil) in S-IV (Table 3). Highest number of AM fungal species were recovered from Abelmoschus 

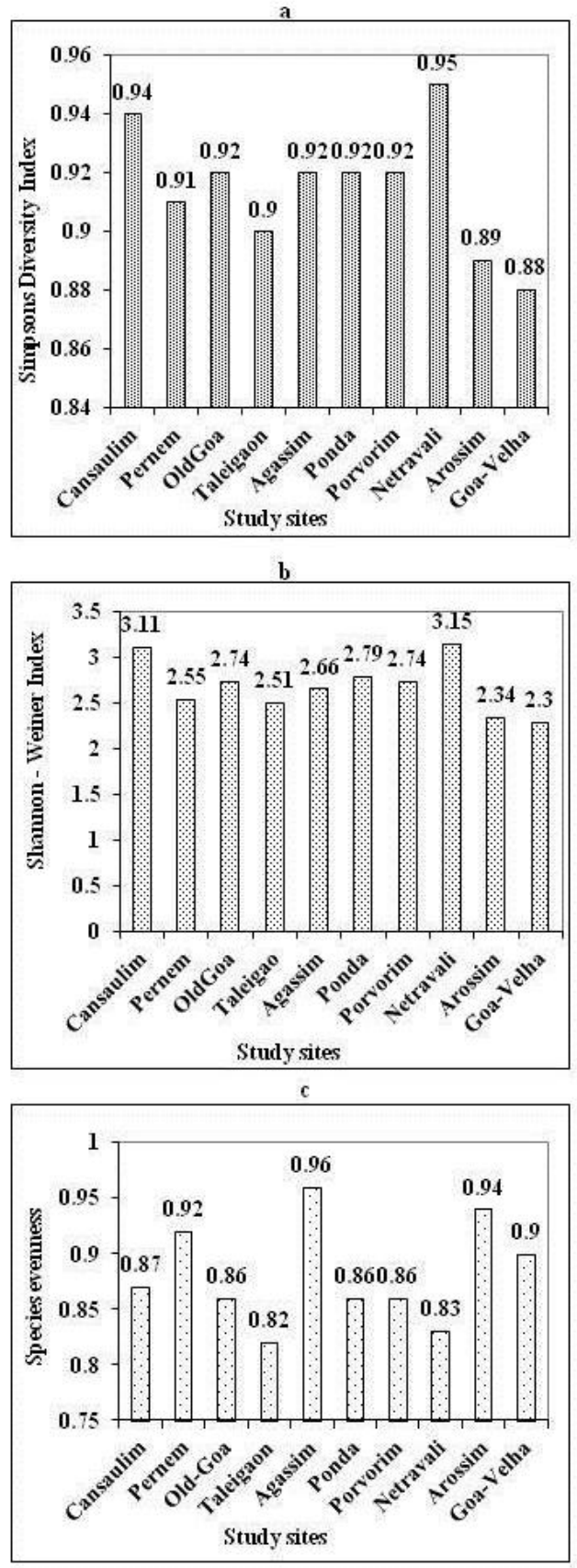

Fig. 2 - AM fungal diversity indices in vegetable crop plants in agricultural sites of Goa a Simpson's Diversity Index b Shannon-Wiener Diversity Index c Species evenness.

esculentus (9) in S-VIII, Cucumis sativus (9) in $\mathrm{S}$-III and $C$. melo (9) in S-VI, and the least number (2) isolated from $A$. viridis, $A$. cruentus, $R$. sativus in $\mathrm{S}-\mathrm{V}, \mathrm{S}-\mathrm{X}$ and $B$. rapa in S-IX (Table 3).

A total of 51 AM fungal species (Fig. 3 $\mathrm{c}$ to $\mathrm{z}$ ) were recorded from ten agricultural sites (Table 3) and Glomus spp. (26) was the dominant genus followed by Acaulospora spp. (16), Gigaspora spp. (4) and Scutellospora spp. (5). Acaulospora appendicula and Glomus albidum were isolated only once in $A$. esculentus from site S-VIII and G. flavisporum was recorded only once in Luffa acutangula in S-III. A. rugosa was reported only in S-IV, in two vegetables, $L$. siceraria and $Z$. mays. Acaulospora scrobiculata was recorded in all sites, in the greatest number of vegetable crops (24) and was dominant in six sites. Acaulospora nicolsonii was also found in all sites in 19 different vegetables.

The abundant AM species recorded were A. scrobiculata in S-I (94), S-II (53), S-V (68), S-VI (87), S-VII (94), S-VIII (58), A. 


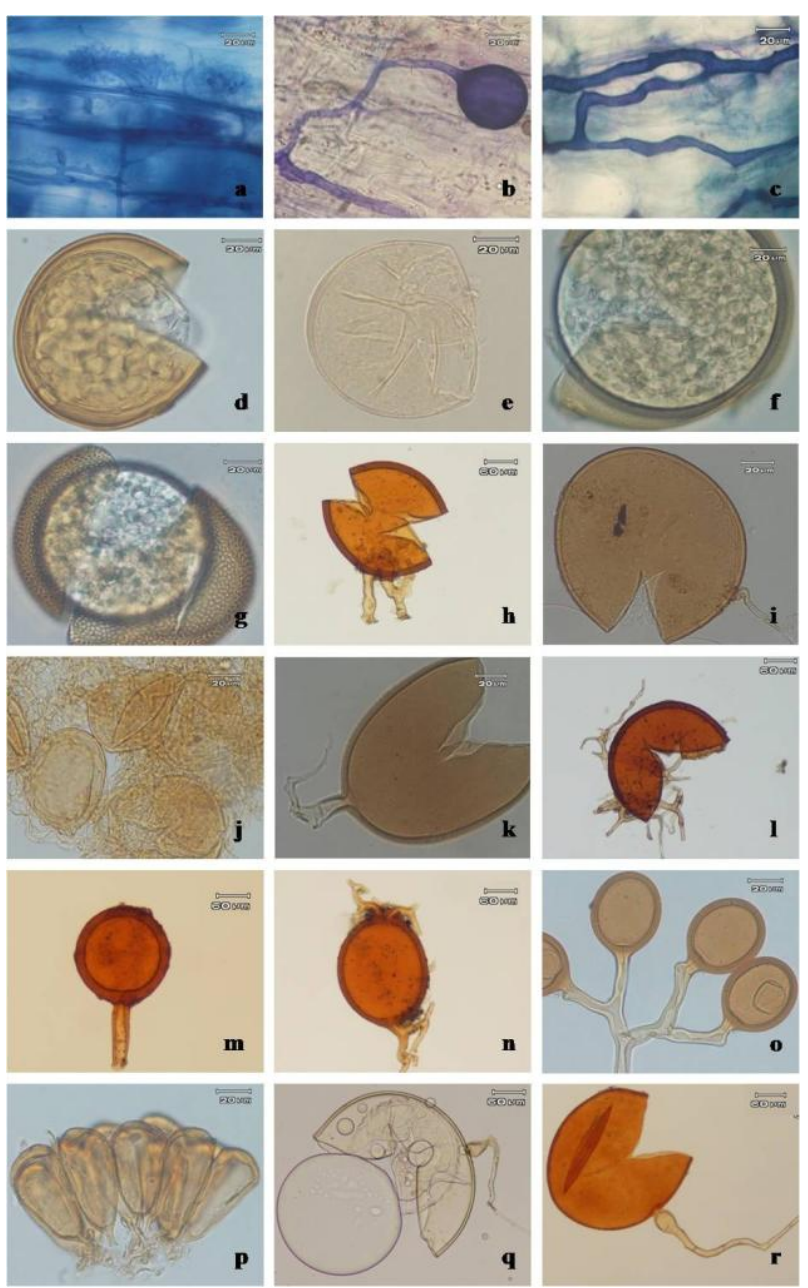

Fig. 3 - AM fungal root colonization and spores isolated from rhizosphere soils of vegetable crop plants. a-c intra-radicle morphological root colonization: arbuscule, vesicle, hypha. d-g Acaulospora species: A. dilatata, A. myriocarpa, A. rehmii, A. scrobiculata. h-p Glomus species: G. ambisporum, G. coremioides, G. constrictum, G. fasciculatum, G. formosanum, G. geosporum, G. multicaule, G. rubiformis, G. taiwanensis. q-r Gigaspora species: Gi. albida, Gi. decipiens.

Table 2 Percent root colonization in vegetable crop plants of Goa.

\begin{tabular}{|c|c|c|c|c|c|c|c|c|c|c|}
\hline Vegetable crops & S-I & S-II & S-III & S-IV & S-V & S-VI & S-VII & S-VIII & S-IX & S-X \\
\hline $\begin{array}{l}\text { Allium cepa } \mathrm{L} . \\
\text { (Liliaceae) }\end{array}$ & $\begin{array}{l}40.00 \\
\pm \\
6.08\end{array}$ & nd & nd & nd & nd & nd & nd & nd & $\begin{array}{c}49.33 \\
\pm \\
7.63\end{array}$ & nd \\
\hline $\begin{array}{l}\text { Amaranthus cruentus } \mathrm{L} . \\
\text { (Amarathaceae) }\end{array}$ & - & - & - & - & - & - & nd & - & - & - \\
\hline $\begin{array}{l}\text { Abelmoschus esculentus } \\
\text { (L.) Moench. } \\
\text { (Malvaceae) }\end{array}$ & $\begin{array}{c}46.33 \\
\pm \\
9.50\end{array}$ & $\begin{array}{c}53.00 \\
\pm \\
5.00\end{array}$ & $\begin{array}{l}41.33 \\
\pm \\
9.45\end{array}$ & $\begin{array}{c}52.67 \\
\pm \\
10.01\end{array}$ & $\begin{array}{l}50.33 \\
\pm \\
14.74\end{array}$ & $\begin{array}{c}57.00 \\
\pm \\
10.58\end{array}$ & nd & $\begin{array}{c}52.33 \\
\pm \\
15.33\end{array}$ & $\begin{array}{c}53.38 \\
\pm \\
13.57\end{array}$ & $\begin{array}{c}56.33 \\
\pm \\
10.40\end{array}$ \\
\hline $\begin{array}{l}\text { Arachis hypogaea } \mathrm{L} \text {. } \\
\text { (Fabaceae) }\end{array}$ & nd & nd & nd & nd & nd & nd & $\begin{array}{c}67.00 \\
\pm \\
11.00\end{array}$ & nd & nd & nd \\
\hline $\begin{array}{l}\text { Amaranthus viridis } \mathrm{L} . \\
\text { (Amarathaceae) }\end{array}$ & - & nd & nd & - & - & - & nd & - & - & - \\
\hline $\begin{array}{l}\text { Brassica rapa } \mathrm{L} \text {. } \\
\text { (Brassicaceae) }\end{array}$ & - & nd & - & - & nd & nd & nd & - & - & - \\
\hline $\begin{array}{l}\text { Capsicum annиит L. } \\
\text { (Solanaceae) }\end{array}$ & $\begin{array}{c}55.33 \\
\pm \\
8.50 \\
\end{array}$ & $\begin{array}{c}44.33 \\
\pm \\
10.50 \\
\end{array}$ & $\begin{array}{c}43.00 \\
\pm \\
7.00 \\
\end{array}$ & $\begin{array}{c}55.67 \\
\pm \\
6.50\end{array}$ & nd & nd & $\begin{array}{c}59.00 \\
\pm \\
13.00 \\
\end{array}$ & $\begin{array}{c}50.33 \\
\pm \\
10.69\end{array}$ & $\begin{array}{c}48.00 \\
\pm \\
12.16\end{array}$ & $\begin{array}{c}54.00 \\
\pm \\
10.00 \\
\end{array}$ \\
\hline
\end{tabular}


Plant Pathology \& Quarantine — Doi 10.5943/ppq/2/2/1

\begin{tabular}{|c|c|c|c|c|c|c|c|c|c|c|}
\hline $\begin{array}{l}\text { Coccinia grandis (L.). } \\
\text { J.Voigt (Cucurbitaceae) }\end{array}$ & $\begin{array}{l}45.33 \\
\pm \\
6.50\end{array}$ & $\begin{array}{c}56.67 \\
\pm \\
10.01\end{array}$ & $\begin{array}{l}54.33 \\
\pm \\
8.50\end{array}$ & $\begin{array}{l}52.00 \\
\pm \\
4.00\end{array}$ & nd & nd & $\begin{array}{l}65.00 \\
\pm \\
9.16\end{array}$ & nd & $\begin{array}{c}49.00 \\
\pm \\
13.45\end{array}$ & nd \\
\hline $\begin{array}{l}\text { Cucumis melo L. } \\
\text { (Cucurbitaceae) }\end{array}$ & $\begin{array}{c}53.00 \\
\pm \\
5.00\end{array}$ & nd & nd & nd & $\begin{array}{c}56.33 \\
\pm \\
9.07\end{array}$ & $\begin{array}{c}46.00 \\
\pm \\
12.00\end{array}$ & nd & nd & nd & nd \\
\hline $\begin{array}{l}\text { Cucurbita moschata } \\
\text { Duchesne ex Poir. } \\
\text { (Cucurbitaceae) }\end{array}$ & $\begin{array}{l}49.67 \\
\pm \\
6.02 \\
\end{array}$ & nd & nd & nd & $\begin{array}{l}57.00 \\
\pm \\
10.14 \\
\end{array}$ & $\begin{array}{c}49.33 \\
\pm \\
8.73 \\
\end{array}$ & nd & nd & nd & nd \\
\hline $\begin{array}{l}\text { Cucurbita pepo } \\
\text { Duchesne. } \\
\text { (Cucurbitaceae) }\end{array}$ & nd & nd & nd & nd & nd & $\begin{array}{l}54.33 \\
\pm \\
12.50 \\
\end{array}$ & nd & nd & nd & $\begin{array}{l}51.00 \\
\pm \\
12.00 \\
\end{array}$ \\
\hline $\begin{array}{l}\text { Cucumis sativus L. } \\
\text { (Cucurbitaceae) }\end{array}$ & $\begin{array}{c}51.67 \\
\pm \\
6.42\end{array}$ & $\begin{array}{l}57.67 \\
\pm \\
13.01 \\
\end{array}$ & $\begin{array}{c}67.33 \\
\pm \frac{}{7} 3 \\
\end{array}$ & nd & nd & $\begin{array}{c}50.66 \\
\pm \\
12.58\end{array}$ & $\begin{array}{c}53.00 \\
\pm \\
14.00\end{array}$ & nd & nd & nd \\
\hline $\begin{array}{l}\text { Cyamopsis } \\
\text { tetragonoloba (L.) Taub. } \\
\text { (Fabaceae) }\end{array}$ & $\begin{array}{c}56.33 \\
\pm \\
4.50\end{array}$ & $\begin{array}{l}61.33 \\
\pm \\
10.21\end{array}$ & $\begin{array}{l}58.33 \\
\pm \\
11.50\end{array}$ & $\begin{array}{l}61.66 \\
\pm \\
10.59\end{array}$ & $\begin{array}{l}58.00 \\
\pm \\
12.28\end{array}$ & $\begin{array}{l}63.67 \\
\pm \\
8.02\end{array}$ & $\begin{array}{c}56.00 \\
\pm \\
11.00\end{array}$ & $\begin{array}{c}56.33 \\
\pm \\
11.01\end{array}$ & $\begin{array}{r}48.51 \\
\pm 8.02\end{array}$ & $\begin{array}{l}57.33 \\
\pm \\
10.06\end{array}$ \\
\hline $\begin{array}{l}\text { Daucus carota } \mathrm{L} . \\
\text { (Apiaceae) }\end{array}$ & nd & nd & nd & $\begin{array}{c}64.67 \\
\pm \\
10.26 \\
\end{array}$ & nd & nd & nd & nd & nd & nd \\
\hline $\begin{array}{l}\text { Ipomoea batatas }(\mathrm{L} .) \\
\text { Lam. (Convolvulaceae) }\end{array}$ & $\begin{array}{l}64.00 \\
\pm \\
6.00 \\
\end{array}$ & nd & nd & $\begin{array}{c}53.00 \\
\pm \\
5.00\end{array}$ & $\begin{array}{c}62.67 \\
\pm \\
15.01 \\
\end{array}$ & nd & nd & $\begin{array}{c}51.00 \\
\pm \\
15.71 \\
\end{array}$ & nd & $\begin{array}{l}58.00 \\
\pm \\
11.00\end{array}$ \\
\hline $\begin{array}{l}\text { Lagenaria siceraria } \\
\text { (Molina) Standl. } \\
\text { (Cucurbitaceae) }\end{array}$ & $\begin{array}{c}51.00 \\
\pm \\
7.21 \\
\end{array}$ & nd & nd & $\begin{array}{l}38.00 \\
\pm \\
12.52\end{array}$ & $\begin{array}{l}52.33 \\
+ \\
14.50\end{array}$ & nd & $\begin{array}{l}52.67 \\
+ \\
14.01\end{array}$ & nd & $\begin{array}{c}36.00 \\
\pm \\
14.52\end{array}$ & $\begin{array}{l}56.33 \\
\pm \\
10.50\end{array}$ \\
\hline $\begin{array}{l}\text { Luffa acutangula (L.) } \\
\text { Roxb. (Cucurbitaceae) }\end{array}$ & nd & nd & $\begin{array}{l}57.67 \\
\pm \\
10.26\end{array}$ & nd & nd & $\begin{array}{c}52.33 \\
\pm \\
13.50\end{array}$ & $\begin{array}{l}56.67 \\
\pm \\
13.05\end{array}$ & nd & nd & nd \\
\hline $\begin{array}{l}\text { Lycopersicon } \\
\text { esculentum Mill. } \\
\text { (Solanaceae) }\end{array}$ & $\begin{array}{l}41.67 \\
\pm \\
8.96 \\
\end{array}$ & nd & nd & nd & nd & nd & $\begin{array}{l}56.33 \\
+ \\
9.45\end{array}$ & nd & $\begin{array}{c}49.00 \\
\pm \stackrel{+}{8} 0 \\
\end{array}$ & nd \\
\hline $\begin{array}{l}\text { Momordica charantia } \\
\text { Descourt. } \\
\text { (Cucurbitaceae) }\end{array}$ & nd & $\begin{array}{l}45.33 \\
\pm \\
9.50 \\
\end{array}$ & $\begin{array}{c}62.00 \\
\pm \\
7.54 \\
\end{array}$ & nd & $\begin{array}{l}57.00 \\
\pm \\
13.00 \\
\end{array}$ & $\begin{array}{l}49.00 \\
\pm \\
6.08 \\
\end{array}$ & $\begin{array}{l}53.00 \\
\pm \\
7.00 \\
\end{array}$ & nd & nd & nd \\
\hline $\begin{array}{l}\text { Raphanus sativus L. } \\
\text { (Brassicaceae) }\end{array}$ & - & nd & nd & - & - & - & - & nd & - & - \\
\hline $\begin{array}{l}\text { Solanum melongena L. } \\
\text { (Solanaceae) }\end{array}$ & $\begin{array}{l}49.33 \\
\pm \\
10.50\end{array}$ & $\begin{array}{l}64.33 \\
\pm \\
10.78\end{array}$ & $\begin{array}{l}61.00 \\
\pm \\
9.16\end{array}$ & $\begin{array}{c}61.67 \\
\pm \\
11.59\end{array}$ & $\begin{array}{l}50.67 \\
\pm \\
8.20\end{array}$ & nd & $\begin{array}{c}62.00 \\
\pm \\
7.54\end{array}$ & $\begin{array}{c}52.00 \\
\pm \\
12.28\end{array}$ & $\begin{array}{c}55.33 \\
\pm \\
11.06\end{array}$ & $\begin{array}{l}52.23 \\
\pm \\
15.04\end{array}$ \\
\hline $\begin{array}{l}\text { Spinacia oleraceae L. } \\
\text { (Amaranthaceae) }\end{array}$ & - & nd & nd & - & - & nd & nd & nd & nd & - \\
\hline $\begin{array}{l}\text { Trichosanthus L. } \\
\text { Cucumerina } \\
\text { (Cucurbitaceae) }\end{array}$ & nd & nd & nd & nd & $\begin{array}{c}53.33 \\
\pm \\
14.04 \\
\end{array}$ & $\begin{array}{c}46.00 \\
\pm \\
10.00\end{array}$ & nd & nd & nd & nd \\
\hline $\begin{array}{l}\text { Trigonella foenum- } \\
\text { graecum L. (Fabaceae) }\end{array}$ & $\begin{array}{c}52.33 \\
\pm \\
7.50 \\
\end{array}$ & nd & nd & $\begin{array}{c}48.33 \\
\pm \\
10.50 \\
\end{array}$ & nd & nd & nd & nd & nd & nd \\
\hline $\begin{array}{l}\text { Vigna sesquipedalis } \\
\text { (L.)Verdc. (Fabaceae) }\end{array}$ & $\begin{array}{l}64.00 \\
\pm \frac{}{6} 00\end{array}$ & $\begin{array}{l}60.00 \\
\pm \\
10.00\end{array}$ & nd & $\begin{array}{c}54.67 \\
\pm \\
10.01\end{array}$ & $\begin{array}{l}57.00 \\
\pm \\
11.53\end{array}$ & $\begin{array}{l}47.00 \\
\pm \\
13.74\end{array}$ & $\begin{array}{l}56.00 \\
\pm \\
10.53\end{array}$ & nd & nd & nd \\
\hline $\begin{array}{l}\text { Vigna unguiculata }(\mathrm{L} .) \\
\text { Walp. } \\
\text { (Fabaceae) }\end{array}$ & $\begin{array}{l}63.00 \\
\pm \frac{ \pm}{6.55}\end{array}$ & nd & nd & $\begin{array}{l}52.00 \\
\pm \\
12.12\end{array}$ & $\begin{array}{l}58.00 \\
\pm \\
9.53\end{array}$ & $\begin{array}{l}55.00 \\
\pm \\
12.16\end{array}$ & $\begin{array}{l}56.33 \\
\pm \\
8.32\end{array}$ & $\begin{array}{c}54.67 \\
\pm \\
12.01\end{array}$ & nd & $\begin{array}{l}46.00 \\
\pm \\
14.52\end{array}$ \\
\hline $\begin{array}{l}\text { Zea mays L. } \\
\text { (Poaceae) }\end{array}$ & $\begin{array}{c}55.33 \\
\pm \\
7.76\end{array}$ & nd & nd & $\begin{array}{c}68.33 \\
+ \\
14.01\end{array}$ & nd & nd & $\begin{array}{c}52.67 \\
+\quad \\
11.37\end{array}$ & nd & nd & nd \\
\hline
\end{tabular}

Legend: - = AM colonization was absent, nd = Vegetable crop plants not detected in the sites, $\pm=$ std.dev. $\mathrm{n}=3 \mathrm{~S}=$ site; S-I = Cansaulim, S-II = Dhargal, S-III = Old-Goa, S-IV = Taleigao, S-V = Agassim, S-VI = Farmagudi, S-VII = Porvorim, S-VIII = Netravali, S-IX = Arossim, S-X = Goa-Velha. 
nicolsonii S-IX (66), A. undulata S-IV (101), G. maculosum S-III (69) and G. manihotis S-X (107) (Table 4).

Species richness was maximum in $\mathrm{S}$ VIII (28 species site ${ }^{-1}$ ) and minimum in S-IX and S-X (11 species site $\left.{ }^{-1}\right)$. Simpson's Diversity Index was high in S-VIII (0.95) (Fig. 2a) and Shannon-Wiener Diversity Index of AM fungi was more in S-VIII (3.15) (Fig. 2b). Species evenness of AM fungal species was high in S-V (0.96) as compared to the other nine sites (Fig. 2c).

\section{Discussion}

Available soil-P was found to be high in the agricultural fields which may be due to frequent fertilization of soils in the field (Mathimaran et al. 2007). Fertilizer management has a direct effect on the performance of AM fungi in a system (Miller \& Jackson 1998) where generally P fertilizer reduces AM fungal colonization and effectiveness, varying with species (Schubert \& Hayman 1986). AM association and spore numbers can also be affected by rapid changes in soil nutrients (Abbott \& Robson 1991). Studies on the increased uptake of $\mathrm{K}, \mathrm{Mg}, \mathrm{Fe}$ and other mineral elements by AM fungi have been reported in the previous studies (Smith \& Read 1997; Clark \& Zeto 2000). Enhancement of $\mathrm{Zn}$ and $\mathrm{Cu}$ uptake by AM plants, attribute to the better uptake and transport in external hyphae to the host plant is also reported (Cumming \& Ning 2003).

AM colonization was absent in family belonging to Amaranthaceae and Brassicaceae. This may be due to the release of root exudate by these two families, (Sanon et al. 2009) which must have lead to the reduced susceptibility of plants to mycorrhization (Tester et al. 1987).

The variations in AM fungal root colonization and spore density may be due to habitat differences, environmental factors, soil fertility, application of fertilizers (Brundrett 1991) or soil disturbance in the sites (Jasper et al. 1991; Boddington \& Dodd 2000). Howeler et al. (1987) also observed large variation in root colonization and spore numbers in rhizosphere soils of crop species grown in different agricultural sites. Therefore $\mathrm{P}$ application rates have to be carefully tuned within the frames of integrated soil fertility management so as not to dramatically decrease continuing benefits of plants from mycorrhizal symbiosis (Mathimaran et al. 2007).

In the present study Glomus spp. (26) was the dominant genus which may be due to the fact that it possesses the ability to germinate in a wide range of temperature and $\mathrm{pH}$ (Wang \& Tschen 1997) followed by Acaulospora spp. (16), Gigaspora spp. (4) and Scutellospora spp. (5). Some of the species, Acaulospora appendicula, A. rugosa, Glomus albidum, G. flavisporum was recorded only once in site and in particular vegetable crops. The rare occurrence of AM fungal species only in one site and/or in particular vegetable crops may be due to the acclimatization of a particular AM fungal genus/species to a particular location (Brundrett 1991).

Acaulospora nicolsonii and Acaulospora scrobiculata was recorded in all sites. Previous studies reported that Acaulospora species are often associated with acidic soils (Morton 1986; Abbott \& Robson 1991).The present study however, shows that Acaulospora species were present in both acid soils ranging from 6.1 to 6.8 and in neutral soils with $\mathrm{pH}$ 7.0. A similar diversity study reported that Acaulospora species were found in alkaline soil with $\mathrm{pH} 7.2$ (Chetan et al. 2008). Wang et al. (1993) found that $\mathrm{pH}$ had little effect on mycorrhizal colonization of oat and potato, but markedly altered the species diversity of the colonizing fungi.

There was variation in abundant AM species recorded in different sites. These variation in spore abundance of different AM fungi in different sites was observed in earlier studies (Schenk \& Kinloch 1980; Chetan et al. 2008). Mathimaran et al. 2007, reported higher spore abundance of Acaulospora species in rotation of Z. mays and Crotolaria spp. in agricultural field.

It has been reported that species richness index is dependent on the sample size, the greater the number of samples collected, the greater the number of species is likely to be recovered (Radhika \& Rodrigues 2010). The variation in AM fungal diversity in rhizosphere soils in the different sites may be due to other factors such as $\mathrm{pH}$, available $\mathrm{P}$ or others nutrients in the soil (Chetan et al. 2008). The 
Table 3 AM fungal species and spore density in agricultural sites of Goa.

\begin{tabular}{|c|c|c|c|c|c|c|c|}
\hline Sites & $\begin{array}{c}\text { Allium } \\
\text { cepa }\end{array}$ & $\begin{array}{c}\text { Amaranthus } \\
\text { cruentus }\end{array}$ & $\begin{array}{c}\text { Amaranthus } \\
\text { esculentus }\end{array}$ & $\begin{array}{c}\text { Arachis } \\
\text { hypogaea }\end{array}$ & $\begin{array}{c}\text { Amaranthus } \\
\text { viridis }\end{array}$ & $\begin{array}{l}\text { Brassica } \\
\text { rapa }\end{array}$ & $\begin{array}{c}\text { Capsicum } \\
\text { annuum }\end{array}$ \\
\hline S-I & $\begin{array}{l}\text { A. di, A. sc, } \\
\text { G. ho } \\
44.33 \pm 1.15\end{array}$ & $\begin{array}{l}\text { A. me, G. cla, } \\
\text { S.bi } \\
16.67 \pm 4.16\end{array}$ & $\begin{array}{l}\text { A. de, A. sc, } \\
\text { G. mac } \\
66.67 \underline{+6.43}\end{array}$ & nd & $\begin{array}{l}\text { A. di, G. } \\
\text { con, G. geo } \\
18.67 \pm 3.21\end{array}$ & $\begin{array}{l}\text { A.di, } \\
\text { A.me, } \quad G . \\
\text { tai } \\
16.33 \pm 1.15\end{array}$ & $\begin{array}{l}\text { A. sc, G. etu, } \\
\text { G. geo, G. glo, } \\
\text { S. bi } \\
73.00 \pm 4.36\end{array}$ \\
\hline S-II & nd & $\begin{array}{l}\text { G. for, G. fas, } \\
\text { G. sin } \\
19.00 \pm 2.65\end{array}$ & $\begin{array}{l}\text { A. sc, } G . \\
\text { cor, G. mos } \\
32.67 \pm 2.52\end{array}$ & nd & nd & nd & $\begin{array}{l}\text { G. mon, } \\
\text { mul, G. tai } \\
45.67 \pm 1.5)\end{array}$ \\
\hline S-III & nd & $\begin{array}{l}\text { A. me, A. un, } \\
\text { G. sin, G. tai } \\
21.00 \pm 1.00\end{array}$ & $\begin{array}{l}\text { A. me, A. fo, } \\
\text { A. sc, G. } \\
\text { mac, G. mul } \\
44.66 \pm 8.52\end{array}$ & nd & nd & $\begin{array}{l}\text { A. di, A. sc, } \\
\text { G. mac, G. } \\
\text { mul, G. tai } \\
20.33 \pm 5.53\end{array}$ & $\begin{array}{l}\text { A. di, A. ni, } \\
\text { G. con, G. for, } \\
\text { G. pac } \\
77.33 \pm 7.02\end{array}$ \\
\hline S-IV & nd & $\begin{array}{l}\text { A. ni, A. my, } \\
\text { A. sc, A. un } \\
17.67 \pm 10.53\end{array}$ & $\begin{array}{l}\text { A. di, A. sp, } \\
\text { A. un, Gi. gi } \\
\text { 56.00+12.65 }\end{array}$ & nd & $\begin{array}{l}\text { A. sp, A. my, } \\
\text { A. un } \\
12.33 \pm 9.52\end{array}$ & nd & $\begin{array}{l}\text { A. sc, A. sp, A. } \\
\text { un, G. sin, Gi. } \\
\text { gi } 57.33 \pm 2.08\end{array}$ \\
\hline S-V & nd & $\begin{array}{l}\text { A. me, G. mic } \\
14.33 \pm 2.08\end{array}$ & $\begin{array}{l}\text { A. ni, A. sc, } \\
\text { G. ge. } \\
21.33 \pm 3.21\end{array}$ & nd & $\begin{array}{l}\text { A. di, A.sc } \\
17.67 \pm 1.53\end{array}$ & nd & nd \\
\hline S-VI & nd & $\begin{array}{l}\text { A. de, A. di, } \\
\text { A. ni, A. sc } \\
30.33 \pm 1.53\end{array}$ & $\begin{array}{l}\text { A. de, A. di, } \\
\text { G. cor } \\
35.67 \pm 4.16\end{array}$ & nd & $\begin{array}{l}\text { A. sc, G. } \\
\text { clav, G. geo } \\
27.00 \pm 2.00\end{array}$ & nd & nd \\
\hline $\begin{array}{c}\text { S- } \\
\text { VII }\end{array}$ & nd & nd & nd & $\begin{array}{l}\text { A. di,A.sc, } \\
\text { G. clav, G. } \\
\text { geo, Gi. gi } \\
76.00 \pm 4.16\end{array}$ & $\begin{array}{l}\text { A. di, A. sc, } \\
\text { G. clav } \\
19.00 \pm 3.61\end{array}$ & nd & $\begin{array}{l}\text { A. di, A. un, } \\
\text { G. geo } \\
30.67 \pm 3.06\end{array}$ \\
\hline $\begin{array}{c}\text { S- } \\
\text { VIII }\end{array}$ & nd & $\begin{array}{l}\text { A. fo, A. sc, G. } \\
\text { mac, G. macu, } \\
\text { G. pac, G. tai, } \\
\text { G. al } \\
23.13 \pm 2.65\end{array}$ & $\begin{array}{l}\text { A. ap, A. } \\
\text { de, A. sc, } \\
\text { G. alb, } \\
\text { G. cal, G. } \\
\text { fas, G. geo, } \\
\text { G. hoi, G. } \\
\text { mac } \\
\text { 58.32 } \pm 3.12\end{array}$ & nd & $\begin{array}{l}\text { A. ni, G. fas, } \\
\text { G.geo, } \\
\text { G.mac, G. } \\
\text { mic, Gi. al, } \\
\text { S. gr } \\
19.00 \pm 2.00\end{array}$ & $\begin{array}{l}\text { A. ni, A. sc, } \\
\text { G. cal, G. } \\
\text { fas, G. geo, } \\
\text { G. mac, S. } \\
\text { gr } \\
18.33 \pm 2.52\end{array}$ & $\begin{array}{l}\text { G. clar, G. fas, } \\
\text { G. glo, } \\
\text { G. hoi, G. mac, } \\
\text { G. mul, } \\
\text { G. mos, S. gr } \\
84.67 \pm 4.16\end{array}$ \\
\hline S-IX & $\begin{array}{l}\text { A. de, A. } \\
\text { sc, G. geo, } \\
\text { G. mac } \\
59.67 \pm 1.53\end{array}$ & $\begin{array}{l}\text { A. ni, G. cor, } \\
\text { G. rub } \\
18.00 \pm 2.65\end{array}$ & $\begin{array}{l}\text { A. me, A. ni, } \\
\text { A. sc } \\
29.67 \pm 2.08\end{array}$ & nd & $\begin{array}{l}\text { A. } s c, A . n i \text {, } \\
\text { G. cor } \\
22.00 \pm 3.00\end{array}$ & $\begin{array}{l}\text { A. de, } G . \\
\text { fas } \\
20.77 \pm 4.73\end{array}$ & $\begin{array}{l}\text { A. sc, G. cor, } \\
\text { G. tai } \\
40.00 \pm 2.00\end{array}$ \\
\hline S-X & nd & $\begin{array}{l}\text { A. ni, A. sc } \\
14.67 \pm 2.51\end{array}$ & $\begin{array}{l}\text { A. me, A. ni, } \\
\text { G. fas, S. gr } \\
58.00 \pm 3.00\end{array}$ & nd & $\begin{array}{l}\text { A. me, G. fas } \\
19.67 \pm 4.50\end{array}$ & $\begin{array}{l}\text { A. ni, G. } \\
\text { geo, } G . \\
\text { mul } \\
16.33 \pm 4.04\end{array}$ & $\begin{array}{l}\text { A. el, A. sc, } \\
\text { G. mul } \\
44.33+4.04\end{array}$ \\
\hline
\end{tabular}

\begin{tabular}{|c|c|c|c|c|c|c|c|}
\hline Sites & $\begin{array}{c}\text { Coccinia } \\
\text { grandis }\end{array}$ & $\begin{array}{l}\text { Cucumis } \\
\text { melo }\end{array}$ & $\begin{array}{l}\text { Cucurbita } \\
\text { moschata }\end{array}$ & $\begin{array}{c}\text { Cucurbita } \\
\text { pepo }\end{array}$ & $\begin{array}{l}\text { Cucums } \\
\text { sativus }\end{array}$ & $\begin{array}{c}\text { Cyamopsis } \\
\text { tetragonolob } \\
a\end{array}$ & Daucus carota \\
\hline S-I & $\begin{array}{l}\text { A. de, G. } \\
\text { cla, G. cor, } \\
\text { G. etu, G. } \\
\text { geo, G.mos } \\
55.00 \pm 1.00\end{array}$ & $\begin{array}{l}\text { A. di, A. ni, G. } \\
\text { fas, G. cor, G. } \\
\text { tai, Gi. dec } \\
82.67 \pm 6.6)\end{array}$ & $\begin{array}{l}\text { A. me, A. } \\
\text { mo, G. mac } \\
50.00 \pm 2.00\end{array}$ & nd & $\begin{array}{l}\text { A. me, A. sc, } \\
\text { G. mac, } \\
\text { G. tai, } \\
58.67 \pm 3.21\end{array}$ & $\begin{array}{l}\text { A. di, Gi. ra, } \\
\text { S. } g r \\
40.00 \pm 8.00\end{array}$ & $\mathrm{nd}$ \\
\hline
\end{tabular}


Plant Pathology \& Quarantine — Doi 10.5943/ppq/2/2/1

\begin{tabular}{|c|c|c|c|c|c|c|c|}
\hline S-II & $\begin{array}{l}\text { A. ni, A.sc, } \\
\text { G. fas, } \\
\text { G.mac, } \\
\text { G. sin, G. } \\
\text { tai } \\
63.67 \pm 1.53\end{array}$ & nd & nd & nd & $\begin{array}{l}\text { A. den, A. } \\
\text { ni, } \quad \text { A. } \\
\text { sc, A. tu, } \\
\text { G. mul, G. } \\
\text { tai } \\
49.00 \pm 1.00\end{array}$ & $\begin{array}{l}\text { A. tu,G.fas, } \\
\text { G. mac } \\
42.33 \pm 1.53\end{array}$ & nd \\
\hline S-III & $\begin{array}{l}\text { A. sc, G. } \\
\text { con, } \\
\text { G. macu, } \\
\text { G. rub } \\
60.33 \pm 1.53\end{array}$ & nd & nd & nd & $\begin{array}{l}\text { A.fo, A. ni, } \\
\text { A. sc, G.con, } \\
\text { G.fas, G. } \\
\text { mul, G. } \\
\text { macu, } \\
\text { G. pac, G. } \\
\text { tai } \\
60.67 \pm 1.53\end{array}$ & $\begin{array}{l}\text { A. de, A. fo, } \\
\text { G. con, } \\
\text { G. geo, G. for } \\
50.33 \pm 4.16\end{array}$ & nd \\
\hline S-IV & $\begin{array}{l}\text { A. my, A. } \\
n i, \\
\text { A. sc, A. un } \\
60.00 \pm 2.00\end{array}$ & nd & nd & nd & nd & 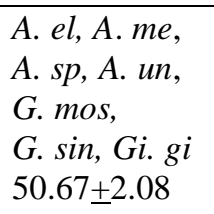 & $\begin{array}{l}\text { A. di, A. me, A. } \\
m y, A . s c, A . \\
s p, A . \text { un } \\
29.67 \pm 1.53\end{array}$ \\
\hline $\mathrm{S}-\mathrm{V}$ & nd & $\begin{array}{l}\text { G. таси, } \\
\text { G. sin, G. tai } \\
49.33 \pm 7.57\end{array}$ & $\begin{array}{l}\text { A. de, G. } \\
m a c, \quad G . \\
m i c \\
56.66 \pm 5.69\end{array}$ & nd & nd & $\begin{array}{l}\text { A. de, A. me, } \\
\text { G. mic } \\
21.33 \pm 2.08\end{array}$ & nd \\
\hline S-VI & nd & $\begin{array}{l}\text { A. ni, A. sc, } \\
\text { G. cor, G. fas, } \\
\text { G. for, } \\
\text { G. macu, G. } \\
\text { tai, G. mul, S. } \\
\text { ni } \\
71.67 \pm 4.93\end{array}$ & $\begin{array}{l}\text { A. el, A. sc, } \\
\text { G. macu } \\
52.67 \pm 1.53\end{array}$ & $\begin{array}{l}\text { A. el, G. } \\
\text { for, } G . \\
\text { geo, } G . \\
\text { macu, } G . \\
\text { tai } \\
59.00 \pm 7.5 \\
0\end{array}$ & $\begin{array}{l}\text { A. di, A. sc, } \\
\text { G. mac } \\
51.33 \pm 3.79\end{array}$ & $\begin{array}{l}\text { G. mul, G. } \\
\text { geo, A. ni } \\
52.33 \pm 2.52\end{array}$ & nd \\
\hline $\begin{array}{c}\text { S- } \\
\text { VII }\end{array}$ & $\begin{array}{l}\text { G. geo, } G . \\
\text { mul, } G . \\
\text { sin, } \\
49.00 \pm 5.10\end{array}$ & nd & nd & nd & $\begin{array}{l}\text { A. sc, A.tu, } \\
\text { G. clav } \\
54.33 \pm 5.03\end{array}$ & $\begin{array}{l}\text { A. de, A. sc, } \\
\text { A. un, G. mul } \\
61.00 \pm 3.61\end{array}$ & nd \\
\hline $\begin{array}{c}\text { S- } \\
\text { VIII }\end{array}$ & nd & nd & nd & nd & nd & $\begin{array}{l}\text { A. sc, G. amb, } \\
\text { G. clar, G. } \\
\text { macu, G. mul, } \\
\text { Gi. al, S. he } \\
72.33 \pm 2.52\end{array}$ & nd \\
\hline S-IX & $\begin{array}{l}\text { A. me, G. } \\
\text { cor, G. geo } \\
26.32 \pm 1.53\end{array}$ & nd & nd & nd & nd & $\begin{array}{l}\text { A. dil, G.geo, } \\
\text { G. maси } \\
42.00 \pm 2.00\end{array}$ & nd \\
\hline S-X & $\overline{\text { nd }}$ & nd & $\begin{array}{l}\text { A. el, A.fo, } \\
\text { A. ni, A. sc, } \\
\text { G. geo, G. } \\
\text { man } \\
66.67 \pm 4.04\end{array}$ & nd & nd & $\begin{array}{l}\text { A. fo, G. geo, } \\
\text { S. gre } \\
59.67 \pm 1.53\end{array}$ & nd \\
\hline
\end{tabular}

\begin{tabular}{|c|c|c|c|c|c|c|c|}
\hline Sites & $\begin{array}{l}\text { Ipomoea } \\
\text { batatas }\end{array}$ & $\begin{array}{c}\text { Lagenaria } \\
\text { siceraria }\end{array}$ & $\begin{array}{c}\text { Lycopersicon } \\
\text { acutangula }\end{array}$ & $\begin{array}{c}\text { Luffa } \\
\text { esculentum }\end{array}$ & $\begin{array}{c}\text { Momordica } \\
\text { charantia }\end{array}$ & $\begin{array}{c}\text { Raphanus } \\
\text { sativus }\end{array}$ & $\begin{array}{c}\text { Solanum } \\
\text { melongena }\end{array}$ \\
\hline S-I & $\begin{array}{l}\text { A. di, A. } \\
\text { sc, G. cla, } \\
\text { G. geo, G. } \\
\text { sin, Gi. de } \\
75.67 \pm 6.11\end{array}$ & $\begin{array}{l}\text { A. sc, G. } \\
\text { cor, G. tai } \\
57.67 \pm 1.52\end{array}$ & nd & $\begin{array}{l}\text { G. cal, S.bi, } \\
\text { S. gr } \\
44.00 \pm 6.56\end{array}$ & nd & $\begin{array}{l}\text { A. me, } G . \\
\text { tai, } S . c a \\
21.33 \pm 3.79\end{array}$ & $\begin{array}{l}\text { A. me, G. etu, G. } \\
\text { macu, S.ca } \\
91.33 \pm 3.21\end{array}$ \\
\hline S-II & nd & nd & nd & nd & $\begin{array}{l}\text { A. } s c, A . s p, \\
\text { G. } m o s \\
36.67 \pm 4.16\end{array}$ & nd & $\begin{array}{l}\text { G. for, G. } \sin , G \text {. } \\
\text { tai } \quad 55.33 \pm 2.30\end{array}$ \\
\hline
\end{tabular}


Plant Pathology \& Quarantine - Doi 10.5943/ppq/2/2/1

\begin{tabular}{|c|c|c|c|c|c|c|c|}
\hline S-III & nd & nd & $\begin{array}{l}\text { A. de, A. fo, } \\
\text { A. sc, G. for, } \\
\text { G. macu, } \\
\text { G. mul, G. } \\
\text { pac, G. fla } \\
79.33+3.79\end{array}$ & nd & $\begin{array}{l}\text { A. me, A. } \\
\text { sc, G. macu } \\
49.00 \pm 2.00\end{array}$ & nd & $\begin{array}{l}\text { A. de, A. me, } \\
\text { G. fas, G. geo } \\
54.33 \pm 2.08\end{array}$ \\
\hline S-IV & $\begin{array}{l}\text { A. me, A. } \\
m y, A . n i, \\
\text { A. re, A. } \\
\text { un, G. mos } \\
29.00 \pm 1.00\end{array}$ & $\begin{array}{l}\text { A. me, A. } \\
\text { ru, A. sp, } \\
\text { A. un } \\
86.67 \pm 0.58\end{array}$ & nd & nd & nd & $\begin{array}{l}\text { A. el, A. my, } \\
\text { A. un } \\
15.33 \pm 3.52\end{array}$ & $\begin{array}{l}\text { A. el, A. me, } \\
\text { A. un, G. sin } \\
42.33 \pm 2.52\end{array}$ \\
\hline S-V & $\begin{array}{l}\text { A. di, A. } \\
\text { sc, } \\
\text { G. clar, } \\
\text { G. mac } \\
76.00 \pm 7.00\end{array}$ & $\begin{array}{l}\text { A. me, A. } \\
\text { sc, } \\
\text { G. fas } \\
45.33+3.51\end{array}$ & nd & nd & $\begin{array}{l}\text { A. di, A. } \\
\text { me, A. sc } \\
30.33 \pm 1.53\end{array}$ & $\begin{array}{l}\text { A. me, } \\
\text { A. } s c \\
18.67 \pm 1.53\end{array}$ & $\begin{array}{l}\text { A. me, A.sc, G. geo, } \\
\text { G. macu } \\
91.00+4.36\end{array}$ \\
\hline S-VI & nd & nd & $\begin{array}{l}\text { A. di, A. sc, } \\
\text { A.sp, G. } \\
\text { amb,G.macu, } \\
\text { S. ni } \\
58.33 \pm 7.64\end{array}$ & nd & $\begin{array}{l}\text { A. ni, A. sc, } \\
\text { G. cor, G. } \\
\text { clav, } G . \\
\text { geo, } G . \\
\text { mac } \\
52.67 \pm 3.05\end{array}$ & $\begin{array}{l}\text { A. di, A. ni, } \\
\text { A.sc, S. gr, } \\
\text { S. ni } \\
16.67 \pm 1.53\end{array}$ & nd \\
\hline $\begin{array}{c}\text { S- } \\
\text { VII }\end{array}$ & nd & $\begin{array}{l}\text { A. me, A. } \\
\text { sc, } \\
\text { G.geo, Gi. } \\
\text { de } \\
25.00 \pm 4.00\end{array}$ & $\begin{array}{l}\text { A. ni, G. } \\
\text { mon, G. sin, } \\
\text { G. tai } \\
63.67 \pm 4.73\end{array}$ & $\begin{array}{l}\text { A. den, A. } \\
\text { un, G. } \\
\text { mul, Gi. de } \\
36.00 \pm 2.65\end{array}$ & $\begin{array}{l}\text { G. clav, } \\
\text { G.cor, Gi. } \\
\text { de } \\
59.33 \pm 2.08\end{array}$ & $\begin{array}{l}\text { A. me, } \\
\text { G.cor, } G . \\
\text { mon } \\
15.27 \pm 1.43\end{array}$ & $\begin{array}{l}\text { A. tu, G. clav, } \\
\text { Gi. gi } \\
32.67 \pm 3.05\end{array}$ \\
\hline $\begin{array}{c}\text { S- } \\
\text { VIII }\end{array}$ & $\begin{array}{l}\text { A. my, A. } \\
\text { sc, G. fas, } \\
\text { G. geo, } \\
\text { Gi. al, } S . \\
\text { he } \\
83.67 \pm 3.73\end{array}$ & nd & nd & nd & nd & nd & $\begin{array}{l}\text { A. den, A. di, A. } \\
\text { me, A. sc, G. pac, } \\
\text { Gi. al, S. gr } \\
78.00 \pm 3.00\end{array}$ \\
\hline S-IX & nd & $\begin{array}{l}\text { A. di, G. } \\
\text { geo, G. tai } \\
73.67 \pm 5.69\end{array}$ & nd & $\begin{array}{l}\text { A. ni, G. } \\
\text { cor, } \quad G . \\
\text { fas } \\
19.00 \pm 2.00\end{array}$ & nd & $\begin{array}{l}\text { A. me, A. ni, } \\
\text { A. sc } \\
14.33 \pm 2.08\end{array}$ & $\begin{array}{l}\text { A. me, A. ni, } \\
\text { G. macu } \\
58.00 \pm 2.00\end{array}$ \\
\hline S-X & $\begin{array}{l}\text { A. el, A. sc, } \\
\text { G. fas, } G . \\
\text { geo, } G . \\
\text { mul } \\
73.67 \pm 3.79\end{array}$ & $\begin{array}{l}\text { A. } s c, G . \\
\text { fas, } \\
\text { S. gr } \\
59.67 \pm 3.15\end{array}$ & nd & nd & nd & $\begin{array}{l}\text { G. fas, G. } \\
\text { mul } \\
13.00 \pm 3.60\end{array}$ & $\begin{array}{l}\text { A. el, A.sc, } \\
\text { geo, G. man } \\
41.00 \pm 3.60\end{array}$ \\
\hline
\end{tabular}

\begin{tabular}{|c|c|c|c|c|c|c|c|}
\hline Sites & $\begin{array}{c}\text { Spinacia } \\
\text { oleraceae }\end{array}$ & $\begin{array}{l}\text { Trichosanthus } \\
\text { cucumerina }\end{array}$ & $\begin{array}{l}\text { Trigonella } \\
\text { foenum- } \\
\text { graecum }\end{array}$ & $\begin{array}{c}\text { Vigna } \\
\text { sesquipedalis }\end{array}$ & $\begin{array}{c}\text { Vigna } \\
\text { unguiculata }\end{array}$ & Zea mays & $\begin{array}{c}\text { Total spore } \\
\text { density }\end{array}$ \\
\hline S-I & $\begin{array}{l}\text { A.me, A. } \\
\text { sc, Gi. ra, } \\
\text { S. ca, S. gr } \\
22.67 \pm 4.16\end{array}$ & nd & $\begin{array}{l}\text { A.sc, Gi. } \\
\text { ra, S. ca } \\
25.67 \pm 3.79\end{array}$ & $\begin{array}{l}\text { A. me, A. sc, } \\
\text { G. clar, G. } \\
\text { macu } \\
57.33 \pm 5.03\end{array}$ & $\begin{array}{l}\text { A. me, A. sc, } \\
\text { Gi. gi, S. gr } \\
68.00 \pm 3.45\end{array}$ & $\begin{array}{l}\text { A. me, A. } \\
\text { sc, G. con, } \\
\text { Gi.de } \\
73.33+5.13\end{array}$ & 1015.01 \\
\hline S-II & nd & nd & nd & $\begin{array}{l}\text { A.sc, G. cor, } \\
\text { G. for, } \\
\text { G.mul, G.sin } \\
49.67 \pm 4.51\end{array}$ & nd & nd & 394.01 \\
\hline S-III & nd & nd & nd & nd & nd & nd & 517.31 \\
\hline
\end{tabular}


Plant Pathology \& Quarantine — Doi 10.5943/ppq/2/2/1

\begin{tabular}{|c|c|c|c|c|c|c|c|}
\hline S-IV & $\begin{array}{l}\text { A. di, A. } \\
m y, \\
\text { A. sc, A. un } \\
27.67 \pm 4.16\end{array}$ & nd & $\begin{array}{l}\text { A. di, A. } \\
\text { me, A. my, } \\
\text { A. sp, A. } \\
\text { un } \\
59.67 \pm 4.51\end{array}$ & $\begin{array}{l}\text { A. me, A. sc, } \\
\text { A. tu, G. sin, } \\
\text { Gi. gi } \\
68.67 \pm 5.51\end{array}$ & nd & $\begin{array}{l}\text { A. di, A. ni, } \\
\text { A. mo, A. } \\
\text { ru, A. un, } \\
\text { G. sin } \\
95.33 \pm 3.51\end{array}$ & 613.01 \\
\hline S-V & $\begin{array}{l}\text { A. sc, } G . \\
\text { macu, } G . \\
\text { mul } \\
23.33 \pm 4.16\end{array}$ & $\begin{array}{l}\text { A. ni, G. mon, } \\
\text { G. sin, G. tai } \\
51.33 \pm 3.21\end{array}$ & nd & $\begin{array}{l}\text { A. sc, G. sin, } \\
\text { G. tai } \\
39.00 \pm 4.00\end{array}$ & $\begin{array}{l}\text { G. mon, } A . \\
\text { dil, } \\
\text { G. fas } \\
56.00 \pm 8.54\end{array}$ & nd & 611.64 \\
\hline S-VI & nd & $\begin{array}{l}\text { A.di, G. for, } \\
\text { G. geo, Gi. al } \\
58.00 \pm 2.00\end{array}$ & nd & $\begin{array}{l}\text { A. sc, G. cor, } \\
\text { G. mul } \\
30.33 \pm 2.52\end{array}$ & $\begin{array}{l}\text { A. de, A. di, } \\
\text { A.ni, G. geo } \\
30.67 \pm 2.08\end{array}$ & nd & 574.34 \\
\hline $\begin{array}{l}\text { S- } \\
\text { VII }\end{array}$ & nd & nd & nd & $\begin{array}{l}\text { A.ni, A.sc, A. } \\
t u, G i . d e \\
24.67 \pm 3.51\end{array}$ & $\begin{array}{l}\text { A. tu, G. } \\
\text { clav, } \\
\text { G. mul,G. } \\
\text { tai } \\
43.00+4.59\end{array}$ & $\begin{array}{l}\text { A. sc, G. } \\
\text { mul, } \quad G i . \\
g i, S . g r \\
52.33 \pm 3.51\end{array}$ & 641.94 \\
\hline $\begin{array}{c}\text { S- } \\
\text { VIII }\end{array}$ & nd & nd & nd & nd & $\begin{array}{l}\text { A. me, A. fo, } \\
\text { A. ni, A. sc, } \\
\text { G. fas, S. } \\
\text { gr, } \quad S . \\
\text { ca, S. he } \\
62.67 \pm 2.08\end{array}$ & nd & 499.8 \\
\hline S-IX & nd & nd & nd & nd & nd & nd & 423.43 \\
\hline S-X & $\begin{array}{l}\text { A. di, A.sc, } \\
\text { G. geo } \\
21.33 \pm 3.21\end{array}$ & nd & nd & nd & $\begin{array}{l}\text { A. sc, G. } \\
\text { geo, G. man } \\
56.33 \pm 6.02\end{array}$ & nd & 544.34 \\
\hline
\end{tabular}

Legend: $\mathrm{S}=$ site; $\mathrm{S}-\mathrm{I}=$ Cansaulim, S-II = Dhargal, S-III = Old-Goa, S-IV = Taleigao, S-V = Agassim, S-VI = Farmagudi, S-VII = Porvorim, S-VIII = Natravali, S-IX = Arossim and S-X = Goa-Velha, nd = Vegetable crops not detected in sites.

AM species : A. ap = Acaulospora appendicula, A. de $=$ A. delicata, A. den $=$ A. denticulata, $A$. di $=$ A. dilatata, A. el $=$ A. elegans, A. fo = A. foveata, A. me = A. mellea, A. my = A. myriocarpa, A. mo = A. morrowiea, A. ni $=A$. nicolsonii, A. re =A. rehmii, A. ru = A. rugosa, A.sp =A. spinosa, A.sc $=$ A. scrobiculata, A. tu $=$ A. tuberculata, A. un $=$ A. undulata,$G$. amb $=$ Glomus ambisporum, G. alb $=$ G. albidum, G. cal $=$ G. caledonium, G. cla $=G$. claroideum, G. clar $=$ G. clarum, G. clav $=$ G. clavispora, .. con $=$ G. constrictum, G. cor $=$ G. coremioides, G. etu $=$ G. etunicatum, G. for $=$ G. formosanum, G. fas $=$ G. fasciculatum, G. fla $=$ G. flavisporum, G. geo = G. geosporum, $G$. glo $=$ G. glomerulatum, G. hoi = G. hoi, G. mac $=$ G. maculosum, G. mac $=$ G. macrocarpum, G. man $=$ G. manihotis, G.mic = G. microcarpum, G.mon = G. monosporum, G. mos $=$ G. mosseae, G. mul = G. multicaule, G. pac $=$ G. pachycaulis, G.rub = G. rubiformis, G.sin = G. sinuosa, G.tai = G. taiwanensis, Gi. al = Gigaspora albida, Gi. $d e=$ Gi. decipiens, Gi. gi = Gi. gigantea, Gi. ra $=$ Gi. ramisporophora, S. bi = Scutellospora biornata, S. ca $=S$. calospora, $S$. gr $=$ S. gregaria, $S$. he $=$ S. heterogama, $S . n i=S$. nigra .

Table 4 Spore abundance of AM fungal species in agricultural sites in Goa.

\begin{tabular}{|l|c|c|c|c|c|c|c|c|c|c|}
\hline \multicolumn{1}{|c|}{ AM species } & S-I & S-II & S-III & S-IV & S-V & S-VI & S-VII & S-VIII & S-IX & S-X \\
\hline A. appendicula & nd & nd & nd & nd & nd & nd & nd & 4 & nd & nd \\
\hline A. delicata & 52 & $\mathrm{nd}$ & 35 & $\mathrm{nd}$ & 23 & 19 & 24 & $\mathrm{nd}$ & 32 & $\mathrm{nd}$ \\
\hline A. denticulata & $\mathrm{nd}$ & 22 & $\mathrm{nd}$ & $\mathrm{nd}$ & $\mathrm{nd}$ & $\mathrm{nd}$ & 19 & 9 & $\mathrm{nd}$ & $\mathrm{nd}$ \\
\hline A. dilatata & 68 & $\mathrm{nd}$ & 23 & 32 & 35 & 52 & 44 & 19 & 42 & 39 \\
\hline A. elegans & $\mathrm{nd}$ & $\mathrm{nd}$ & $\mathrm{nd}$ & 28 & $\mathrm{nd}$ & 16 & $\mathrm{nd}$ & $\mathrm{nd}$ & $\mathrm{nd}$ & 46 \\
\hline A. foveata & $\mathrm{nd}$ & $\mathrm{nd}$ & 29 & $\mathrm{nd}$ & $\mathrm{nd}$ & $\mathrm{nd}$ & $\mathrm{nd}$ & 28 & $\mathrm{nd}$ & 32 \\
\hline A. mellea & 83 & $\mathrm{nd}$ & 32 & 36 & 53 & $\mathrm{nd}$ & 23 & 26 & 33 & 23 \\
\hline A. morrowiea & 28 & $\mathrm{nd}$ & $\mathrm{nd}$ & 9 & $\mathrm{nd}$ & $\mathrm{nd}$ & $\mathrm{nd}$ & $\mathrm{nd}$ & $\mathrm{nd}$ & $\mathrm{nd}$ \\
\hline A. myriocarpa & $\mathrm{nd}$ & $\mathrm{nd}$ & $\mathrm{nd}$ & 88 & $\mathrm{nd}$ & $\mathrm{nd}$ & $\mathrm{nd}$ & 34 & $\mathrm{nd}$ & $\mathrm{nd}$ \\
\hline
\end{tabular}


Plant Pathology \& Quarantine - Doi 10.5943/ppq/2/2/1

\begin{tabular}{|c|c|c|c|c|c|c|c|c|c|c|}
\hline A. nicolsonii & 34 & 27 & 19 & 34 & 23 & 43 & 32 & 24 & 66 & 32 \\
\hline A. rehmii & nd & nd & nd & 29 & nd & nd & nd & nd & nd & nd \\
\hline A. rugosa & nd & nd & nd & 12 & nd & nd & nd & nd & nd & nd \\
\hline A. scrobiculata & 94 & 53 & 32 & 24 & 68 & 87 & 94 & 58 & 37 & 77 \\
\hline A. spinosa & nd & 14 & nd & 38 & nd & 22 & nd & nd & nd & nd \\
\hline A. tuberculata & nd & 19 & nd & 15 & nd & nd & 21 & nd & nd & nd \\
\hline A. undulata & nd & nd & 12 & 101 & nd & nd & 36 & nd & nd & nd \\
\hline G. ambisporum & nd & nd & nd & nd & nd & 12 & nd & 5 & nd & nd \\
\hline G. albidum & nd & nd & nd & nd & nd & nd & nd & 6 & nd & nd \\
\hline G. caledonium & 35 & nd & nd & nd & nd & nd & nd & 14 & nd & nd \\
\hline G. claroideum & 49 & nd & nd & nd & 34 & nd & nd & 24 & nd & nd \\
\hline G. clarum & 19 & nd & nd & nd & nd & nd & nd & 8 & nd & nd \\
\hline G. clavispora & nd & nd & nd & nd & nd & 27 & 52 & nd & nd & nd \\
\hline G.constrictum & 31 & nd & 39 & nd & nd & nd & nd & nd & nd & nd \\
\hline G. coremioides & 30 & 37 & nd & nd & nd & 44 & 19 & nd & 54 & nd \\
\hline G. etunicatum & 42 & nd & nd & nd & nd & nd & nd & nd & nd & nd \\
\hline G. fasciculatum & 37 & 29 & 54 & nd & 46 & 18 & nd & 20 & 33 & 52 \\
\hline G. flavisporum & nd & nd & 6 & nd & nd & nd & nd & nd & nd & nd \\
\hline G. formosanum & nd & 22 & 12 & nd & nd & 33 & nd & nd & nd & nd \\
\hline G. geosporum & 28 & nd & 33 & nd & 35 & 45 & 46 & 22 & 42 & 59 \\
\hline G. glomerulatum & 22 & nd & nd & nd & nd & nd & nd & 9 & nd & nd \\
\hline G. hoi & 13 & nd & nd & nd & nd & nd & nd & 8 & nd & nd \\
\hline G. macrocarpum & nd & 22 & nd & nd & 44 & 15 & nd & 10 & nd & nd \\
\hline G. maculosum & 45 & nd & 69 & nd & 46 & 29 & nd & 19 & 41 & nd \\
\hline G. manihotis & nd & nd & nd & nd & nd & nd & nd & nd & nd & 107 \\
\hline G. microcarpum & nd & nd & nd & nd & 53 & nd & nd & 11 & nd & nd \\
\hline G. monosporum & nd & 9 & nd & nd & 34 & nd & 10 & nd & nd & nd \\
\hline G. mosseae & 78 & 33 & nd & 42 & nd & nd & nd & 19 & nd & nd \\
\hline G. multicaule & nd & 28 & 49 & nd & 32 & 31 & 57 & 23 & nd & 45 \\
\hline G. pachycaulis & nd & nd & 18 & nd & nd & nd & nd & 9 & nd & nd \\
\hline G. rubiformis & nd & nd & 22 & nd & nd & nd & nd & nd & 18 & nd \\
\hline G. sinuosa & 38 & 38 & 17 & 43 & 46 & nd & 24 & nd & nd & nd \\
\hline G. taiwanensis & 55 & 41 & 16 & nd & 39 & 21 & 21 & 22 & 25 & nd \\
\hline Gi. albida & nd & nd & nd & nd & nd & 12 & nd & 23 & nd & nd \\
\hline Gi. decipiens & 33 & nd & nd & nd & nd & nd & 67 & nd & nd & nd \\
\hline Gi. gigantea & 26 & nd & nd & 82 & nd & nd & 34 & nd & nd & nd \\
\hline $\begin{array}{l}\text { Gi. } \\
\text { ramisporophora }\end{array}$ & 8 & nd & nd & nd & nd & nd & nd & nd & nd & nd \\
\hline S. biornata & 26 & nd & nd & nd & nd & nd & nd & nd & nd & nd \\
\hline S. calospora & 16 & nd & nd & nd & nd & nd & nd & 12 & nd & nd \\
\hline S. gregaria & 25 & nd & nd & nd & nd & 17 & 19 & 27 & nd & 32 \\
\hline S. heterogama & nd & nd & nd & nd & nd & nd & nd & 7 & nd & nd \\
\hline S. nigra & nd & nd & nd & nd & nd & 31 & nd & nd & nd & nd \\
\hline
\end{tabular}

Legend: A - Acaulospora, G - Glomus, Gi - Gigaspora, $S$ - Scutellospora, nd = AM species not detected in sites

$\mathrm{S}=$ site; $\mathrm{S}-\mathrm{I}=$ Cansaulim, S-II = Dhargal, S-III = Old-Goa, $\mathrm{S}-\mathrm{IV}=$ Taleigao, $\mathrm{S}-\mathrm{V}=$ Agassim, $\mathrm{S}-\mathrm{VI}=\mathrm{Farmagudi}, \mathrm{S}-\mathrm{VII}$ = Porvorim, S-VIII = Netravali, S-IX = Arossim, S-X = Goa-Velha. 
effectiveness of AM fungal association can be influenced by the crop history of the field, and by crop cultivar (Hendrix et al. 1995).

The AM fungal diversity shown in this study relates to sustainability of an agroecosystem (Sieverding 1990). The greater the diversity, the more benefits conferred to the crops, as the mycorrhizal community will span a broader range of functions (Koide 2000). To optimize management of AM fungi in field conditions there is a need for more information on how agricultural practices influence the variation in AM fungal community development and function in different vegetable crop species. It is necessary to identify AM fungal species in agricultural sites thereby determining the effects of agricultural treatments upon AM fungi and the eventual development of management regimes for these fungi.

\section{Acknowledgements}

Authors gratefully acknowledge the financial assistance received from the Planning Commission, Government of India, New Delhi to carry out this study.

\section{References}

Abbott LK, Robson AD. 1991 - Factors influencing the occurrence of vesicular arbuscular mycorrhiza. Agriculture Ecosystems and Environment 35, 121150.

Almeida RT, Schenck NC. 1990 - A revision of the genus Sclerocyctis (Glomaceae, Glomales). Mycologia 82, 703-714.

Berger KC, Truog E. 1939 - Boron determination in soils and plants. Industrial and Engineering Chemistry, Analytical Edition 11, 540-545.

Boddington CL, Dodd JC. 2000 - The effect of agricultural practices on the development of indigenous arbuscular mycorrhizal fungi. I. Field studies in an Indonesian ultisol. Plant and Soil 218, 137-144.

Brundrett MC. 1991 - Mycorrhiza's in natural ecosystems. Advances in Ecological Research 2, 171-313.

Blaszkowski J. 1994 - Comparative studies on the occurrence of Arbuscular fungi and Mycorrhizae (Glomales) in cultivated and uncultivated soils of Poland. Acta Mycologia 28, 93-140.

Bray RH, Kurtz LT. 1945 - Determination of total, organic, and available forms of phosphorus in soils. Soil Science 59, 3945.

Chetan KKV, Chandrashekar KR, Lakshmipathy R. 2008 - Variation in Arbuscular mycorrhizal fungi and phosphatase activity associated with Sida cordifolia in Karnataka. World Journal of Agricultural Sciences 4, 770-774.

Cumming JR, Ning J. 2003 - Arbuscular mycorrhizal fungi enhance aluminium resistance of broomsedge (Andropogon virginicus L.). Journal of Experimental Botany 54, 1447-1459.

Clark RB, Zeto SK. 2000 - Mineral acquisition by arbuscular mycorrhizal plants. Journal of Plant Nutrition 23, 867-902.

Douds DD, Nagahashil G, Pfeffer PE, Kayser WM, Reider C. 2005 - On-Farm production and utilization of arbuscular mycorrhizal fungus inoculum. Canadian Journal of Plant Sciences 85, 15-21.

Gerdemann JW, Nicolson TH. 1963 - Spore density of Endogone species extracted from soil wet sieving and decanting. Transactions of British Mycological Society 46, 235-244.

George E. 2000 - Nutrient uptake. In: Arbuscular Mycorrhizas: Physiology and Function (eds Y Kappulnick, DD Douds). Kluwer Academic Publication, Netherland 307-344.

Giovanetti M, Mosse B. 1980 - An evaluation of techniques for measuring vesicular arbuscular mycorrhizal infection in roots. New Phytologist 84, 489-500.

Hammer Ø, Harper DAT, Ryan PD. 2001 PAST: Paleontological Statistics Software Package for Education and Data Analysis. Palaeontologia Electronica 4, 9.

Hanway JJ, Heidal H. 1952 - Soil analysis method as used in Iowa State College Soil Testing Laboratory. Iowa Agriculture 57, 1-31.

Harrier LA, Watson CA. 2004 - The potential role of arbuscular mycorrhizal fungi in the bioprotection of plants against soilborne pathogens in organic and/or other 
sustainable farming systems. Pest Management Science 60, 149-157.

Hendrix JW, Guo BZ, AN ZQ. 1995 Divergence of mycorrhizal fungal communities in crop production systems. In: The Significance and Regulation of Biodiversity (eds HP Collins, GP Robertson, MJ Klug). Kluwer Academic Publication, Netherland 131-140.

Howeler RH, Seiverding E, Saif S. 1987 Practical aspects of mycorrhizal technology in some tropical crops and pastures. Plant and Soil 100, 249-283.

Jasper DA, Abbot LK, Robson AD. 1991 - The effect of soil distribution on vesiculararbuscular mycorrhizal fungi in soils from different vegetation types. New Phytologist 118, 471-476.

Khalil S, Loynachan TE, McNabb HS. 1992 Colonization of soybean by mycorrhizal fungi and spore populations in Iowa soils. Agronomy Journal 84, 832-836.

Koide RT. 2000 - Functional complimentarity in the arbuscular mycorrhizal symbiosis. New Phytologist 147, 233-235.

Koske RE, Gemma JN. 1989 - A modified procedure for staining roots to detect VA mycorrhizas. Mycological Research 92, 486-505.

Lindsay WL, Norwell WA. 1978 Development of DTPA soil test for zinc, iron, manganese and copper. Soil Science Society of America Journal 42, 421-428.

Mathimaran N, Ruh R, Jama B, Verchot L, Frossard E, Jansa J. 2007 - Impact of agricultural management on arbuscular mycorrhizal fungal communities in Kenyan ferralsol. Agriculture, Ecosystems and Environment 119, 22-32.

Miller RL and Jackson LE. 1998 - Survey of vesicular-arbuscular mycorrhizae in lettuce production in relation to management and soil factors. Journal of Agricultural Science, Cambridge 130, 173-182.

Morton JB, Benny BL. 1990 - Revised classification of arbuscular mycorrhizal fungi (Zygomycetes): a new order, Glomales, two new suborders Glominae and Gigasporinae and two new families Acaulosporaceae and Gigasporaceae, with an emendation of Glomaceae.
Mycotaxon, 37, 471-491.

Morton JB. 1986 - Three new species of Acaulospora (Endogonaceae) from highaluminium, low $\mathrm{pH}$ soils in West Virginia. Mycologia 78, 641-648.

Pojo MJ, Azcon AC. 2007 - Unraveling mycorrhiza-induced resistance. Current Opinion in Plant Biology 10, 393-398.

Radhika KP, Rodrigues BF. 2010 - Arbuscular mycorrhizal fungal diversity in some commonly occurring medicinal plants of Western Ghats, Goa region. Journal of Forestry Research 21, 45-52.

Rezaee DY, Goltapeh ME, Alizadeh A, Varma A, Mukerji KG. 2007 - Arbuscularmycorrhizal fungi associated with Alfalfa rhizosphere in Iran. American-Eurasian Journal of Agricultural and Environmental Science 2, 574-580.

Rivera BF, Tuinen D, Martin LF, Metwally A, Dietz KJ, Gianinazzi-Pearson V. 2005 Molecular changes in Pisum sativum L. roots during arbuscular mycorrhiza buffering of cadmium stress. Mycorrhiza $16,51-60$.

Rodrigues BF, Muthukumar T. 2009 Arbuscular mycorrhizae of Goa - a manual of identification Protocols, Goa University, Goa 109-135.

Sanon A, Beguiristain T, Cebron A, Berthelin J, Ndoye I, Leyval C, Sylla S, Duponnois R. 2009 - Changes in soil diversity and global activities following invasions of the exotic invasive plant, Amaranthus viridis $\mathrm{L}$. decrease the growth of native Sahelian Acacia species. FEMS Microbiology Ecology 70, 118-131.

Schubert A, Hayman DS. 1986 - Plant growth responses to vesicular- arbuscular mcorrhiza. XVI. Effectiveness of different endophytes at different levels of soil phosphate. New Phytologist 103, 79-90.

Schenk NC, Kinloch RA. 1980 - Incidence of mycorrhizal fungi on six field crops in monoculture on a newly cleared woodland sites Mycologia 72, 445-456.

Schenk NC, Perez Y. 1990 - Manual for identification of VA Mycorrhizal fungi. INVAM, Florida University, Gainesville, USA.

Sieverding E. 1990 - Ecology of VAM fungi in tropical agrosystems. Agriculture, Eco- 
systems and Environment 29, 169-180.

Smith SE, Read DJ. 1997 - Mycorrhizal Symbiosis. 2nd edn. UK Academic Press, London 1-470.

Smith SE, Smith FA, Jakobsen I. 2003 Mycorrhizal fungi can dominate phosphate supply to plants irrespective of growth responses. Plant Physiology 133, 16-20.

Tester M, Smith SE, Smith FA. 1987 - The phenomenon of non-mycorrhizal plants. Canadian Journal of Botany 65, 419-431.

Walkley A, Black JA. 1934 - An examination of the Degtjareff method for determining soil organic matter and a proposed modification of the chromic titration method. Soil Science 37, 29-38.

Wang GM, Stribley DP, Tinker PB, Walker C.
1993 - Effects of $\mathrm{pH}$ on arbuscular mycorrhiza I. Field observations on the long-term liming experiments at Rothamsted and Woburn. New Phytologist 124, 465-472.

Wang C L, Tschen JSM. 1997 - Factors on the spore germination of arbuscular mycorrhizal fungi, Glomus spp. Fungal Science 12, 3-4.

Wang B, Qiu YL. 2006 - Phylogenetic distribution and evolution of mycorrhizas in land plants. Mycorrhiza 16, 299-363.

Yaseen T, Burni T, Hussain F. 2011 - Effect of arbuscular mycorrhizal inoculation on nutrient uptake, growth and productivity of cowpea (Vigna unguiculata) varieties. African Journal of Biotechnology 10, 8593-8598. 